\title{
Validation of a synoptic solar wind model
}

\author{
O. Cohen, ${ }^{1}$ I. V. Sokolov, ${ }^{1}$ I. I. Roussev, ${ }^{2}$ and T. I. Gombosi ${ }^{1}$ \\ Received 5 September 2007; revised 17 October 2007; accepted 21 December 2007; published 19 March 2008.
}

[1] We present a validation of a three-dimensional magnetohydrodynamic model for the solar corona and the inner heliosphere. We compare the results of the model with long-term satellite data at $1 \mathrm{AU}$ for a 1 year period during solar minimum and another year period of solar maximum. Overall, the model predicts rather well the magnitude of the magnetohydrodynamical variables for solar minimum conditions. For solar maximum conditions, the magnitude of the magnetic field predicted by the model is too low. This result is consistent with the assumption that during solar maximum, a significant portion of the heliospheric open magnetic flux is not captured by the magnetogram input and the potential field approximation.

Citation: Cohen, O., I. V. Sokolov, I. I. Roussev, and T. I. Gombosi (2008), Validation of a synoptic solar wind model, J. Geophys. Res., 113, A03104, doi:10.1029/2007JA012797.

\section{Introduction}

[2] The ability to predict on a daily basis the physical conditions in interplanetary space, as well as at the vicinity of the Earth, has become significantly important in the past few decades. Growing dependency on satellite communication, planned space missions, and hazards to systems on the ground has brought the concept of space weather (SW), which is monitored by the Space Weather Prediction Center (formerly called the Space Environment Center) (see more details at www.swpc.noaa.gov) to the fore. Unlike atmospheric forecasting tools, which have been in use for decades, the tools for SW forecasting are still very limited. The prediction of SW depends mostly on satellites which observe activity on the Sun (such as SOHO and STEREO) or satellites which can measure the solar wind conditions at the vicinity of the Earth (such as ACE and Wind). Since the forecasting time of these satellites is about $60 \mathrm{~min}$ [ Gleisner and Watermann, 2006], we need to develop dynamic numerical models for the space environment in order to improve the forecasting time.

[3] The Space Weather Modeling Framework (SWMF) is a computational tool for simulating the space environment from the solar photosphere all the way to the Earth's upper atmosphere. A complete description of the SWMF is given by Tóth et al. [2005]. The SWMF includes the numerical models for the solar corona (SC) and the inner heliosphere (IH), which describe the interplanetary space between the Sun and the Earth. The SC and IH components are used to describe the physical conditions of the ambient solar wind

\footnotetext{
${ }^{1}$ Department of Atmospheric, Oceanic, and Space Sciences, University of Michigan, Ann Arbor, Michigan, USA.

${ }^{2}$ Institute for Astronomy, University of Hawai'i at Manoa, Honolulu, Hawaii, USA.
}

Copyright 2008 by the American Geophysical Union. 0148-0227/08/2007JA012797 and the dynamical conditions during a space weather event [Cohen et al., 2007].

[4] In this paper, we focus on modeling the ambient conditions for the $\mathrm{SC}$ and $\mathrm{IH}$. Since arrival time of the interplanetary shock of a coronal mass ejection (CME) is one of the most important parameters in SW forecasting (others are $B_{z}$, dynamic pressure, etc.), it is important to obtain the correct background conditions through which this shock propagates. Previous work has been done to model the SC and IH using global MHD models. Usmanov [1993], Mikic et al. [1999], Suess et al. [1999], and Groth et al. [2000], for example, used an empirical heating source term in the energy equation to obtain the distribution of the steady state solar wind. Other models [Usmanov et al., 2000; Usmanov and Goldstein, 2003] used a WKB approximation of the Alfvén wave turbulence to obtain the extra energy required to heat and accelerate the solar wind. Wu et al. [1999] and Roussev et al. [2003] assumed that the extra energy gained by the solar wind can be represented as a change in the polytropic index, $\gamma$. The acceleration of the solar wind, as well as the difference in the final speed of the fast and slow solar wind, is obtained by using a nonuniform distribution of $\gamma$ in the energy equation. Our SC model is based on the model by Roussev et al. [2003] and is described in detail by Cohen et al. [2007].

[5] In order to validate a global model, one needs to thoroughly compare its output with observations. For the particular purpose of SW forecasting, the model should match the observations of the spacecraft, which measure the solar wind conditions at the vicinity of the Earth. Here we present a long-term validation of our steady state $\mathrm{SC}$ and $\mathrm{IH}$ model. We compare the model's result with ACE and Wind observations for the hydrodynamical parameters, as well as for the magnetic field. This validation is crucial in order to decide whether the model can be used as an operational SW forecasting system.

[6] We describe the model in section 2, present the results in section 3 , and discuss the main issues of the model in 
$r=1 R_{\odot}:$ $\gamma(\theta, \phi)$ is specified using the Bernoulli Integral.

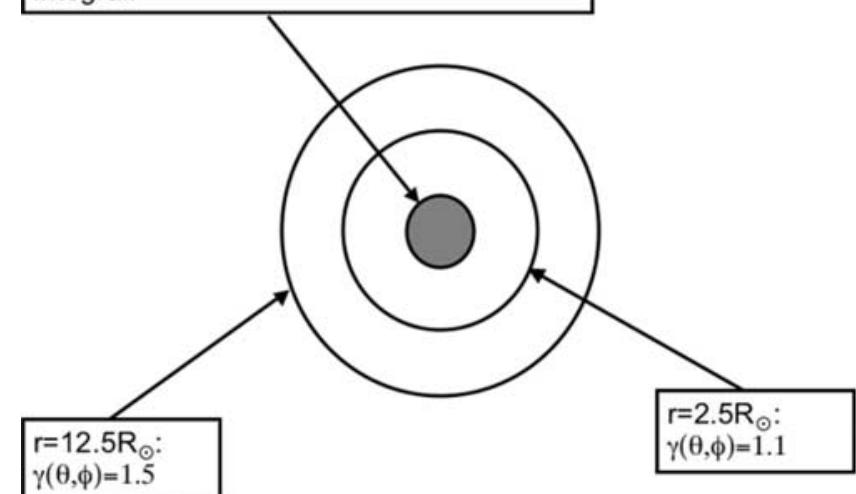

Figure 1. The spatial distribution of $\gamma$ used in our model. Here $\gamma$ is specified on the solar surface using the Bernoulli integral and has been interpolated to a spherically uniform value of 1.1 at $r=2.5 R_{\odot} ; \gamma$ is linearly varied to a value of 1.5 for $2.5 R_{\odot}<r \leq 12.5 R_{\odot}$.

section 4. We draw our conclusions regarding the operational capabilities of the model in section 5 .

\section{MHD Model}

[7] We use the model by Cohen et al. [2007] to obtain the steady state $\mathrm{SC}$ and $\mathrm{IH}$ solution. The $\mathrm{SC}$ and $\mathrm{IH}$ modules of SWMF are versions of the BASTRUS global MHD code, which is fully parallel and has adaptive mesh refinement capabilities [see Powell et al., 1999]. Our SC model is driven by high-resolution SOHO Michelson Doppler Imager (MDI) magnetograms (seehttp://soi.stanford.edu). We use the magnetogram data to calculate the potential magnetic field, assuming the source surface is at $R_{S S}=2.5 R_{\odot}$, where $R_{\odot}$ is the solar radius, and use this distribution of the magnetic field as an initial condition for subsequent relaxation.

[8] The heating and acceleration of the solar wind plasma are achieved by using a nonuniform spatial distribution of $\gamma$. In order to obtain a more realistic distribution, we use the empirical Wang-Sheeley-Arge (WSA) model as an input to our model [Arge and Pizzo, 2000]. The WSA model is found to be very reliable, although, in principle, our model can use any empirical prediction for the solar wind speed, such as that given by Fisk [2003]. The WSA model uses the potential field distribution to obtain the magnetic flux tube expansion factor defined as [Wang and Sheeley, 1990]

$$
f_{s}=\frac{\left|B\left(R_{s s}\right)\right| R_{s s}^{2}}{\left|B\left(R_{\odot}\right)\right| R_{\odot}^{2}} .
$$

[9] The WSA model provides an empirical relation for the distribution of the solar wind speed on a spherical surface at $1 \mathrm{AU}$ as a function of $f_{s}$ and the angular distance of a magnetic field foot point from the coronal hole boundary, $\theta_{b}$. In our model, we use the following formula [Arge et al., 2004]:

$$
\left.u_{s w}=265+\frac{1.5}{\left(1+f_{s}\right)^{1 / 3}}\left\{5.9-1.5 e^{\left[1-\left(\theta_{b} / 7\right)^{5 / 2}\right.}\right]\right\}^{7 / 2} \mathrm{~km} \mathrm{~s}^{-1} .
$$

[10] We assume that far from the Sun the total energy is dominated by the energy of the bulk motion and that the thermal and gravitational energy are negligible. We also assume that at the coronal base the bulk kinetic energy is zero. Owing to energy conservation, we can use the Bernoulli integral (BI) to relate the two ends of a streamline (or magnetic field line):

$$
\frac{u_{s w}^{2}(\theta, \phi)}{2}=\frac{\gamma_{0}\left(\theta_{0}, \phi_{0}\right)}{\left[\gamma_{0}\left(\theta_{0}, \phi_{0}\right)-1\right]} \frac{p_{0}\left(\theta_{0}, \phi_{0}\right)}{\rho_{0}\left(\theta_{0}, \phi_{0}\right)}-\frac{G M_{\odot}}{R_{\odot}} .
$$

[11] Here $u_{s w}$ is the input solar wind speed from the WSA model at $2.5 R_{\odot}, G$ is the gravitational constant, and $M_{\odot}$ is the solar mass; $\gamma_{0}, p_{0}$, and $\rho_{0}$ are the values at the coronal base for the polytropic index, pressure, and mass density, respectively. The coordinates $\theta_{0}, \phi_{0}$ represent the location of the field line foot point, in which $u_{s w}(\theta, \phi)$ originated. We interpolate $\gamma$ from its photospheric value to a spherically uniform value of 1.1 on the source surface at $r=2.5 R_{\odot}$. Here $\gamma$ is linearly varied from 1.1 to 1.5 for $2.54 R_{\odot}<r<$ $12.5 R_{\odot}$, and $\gamma=1.5$ above $12.5 R_{\odot}$ [Totten et al., 1996]. Figure 1 shows the spatial distribution of $\gamma$. Once the spatial distribution of $\gamma$ is obtained, we solve the MHD equations self-consistently using this distribution in the energy equation to obtain the steady state solution for the SC and solar wind.

[12] The above distribution of $\gamma$ enables us to reproduce the bimodal structure of the solar wind speed with relatively sharp transition between the fast wind at higher latitudes and the slow wind at lower latitudes. However, the distributions of the coronal density and temperature are still not determined. It is well established that the fast solar wind originates from coronal holes [ Phillips et al., 1995], where the density and temperature are lower than the density and temperature at the closed field regions. That is why we scale the base density, $\rho_{0}$, and the base temperature, $T_{0}$, at each point on the solar surface with the conjugated value of the input speed from the WSA model (in other words, the base density and temperature are also a function of the expansion factor). We set the velocity to zero on the inner boundary. However, we set the boundary condition for the density to allow finite numerical flux to flow through the inner boundary. We would like our model to be driven only by the magnetogram data without any particular parameterization for each Carrington rotation (CR). Therefore, we parameterize the model for the general cases of solar minimum and solar maximum conditions.

[13] We use nine levels of grid refinement in the SC module to obtain a grid cell size of $0.024 R_{\odot}$ on the solar surface. We refine the grid throughout the simulation using the criteria of field line reversal to obtain a grid cell size of $0.2 R_{\odot}$ near the current sheet. We run the $\mathrm{SC}$ module without the IH module until a steady state is obtained, and we use the local time stepping algorithm to accelerate convergence. 


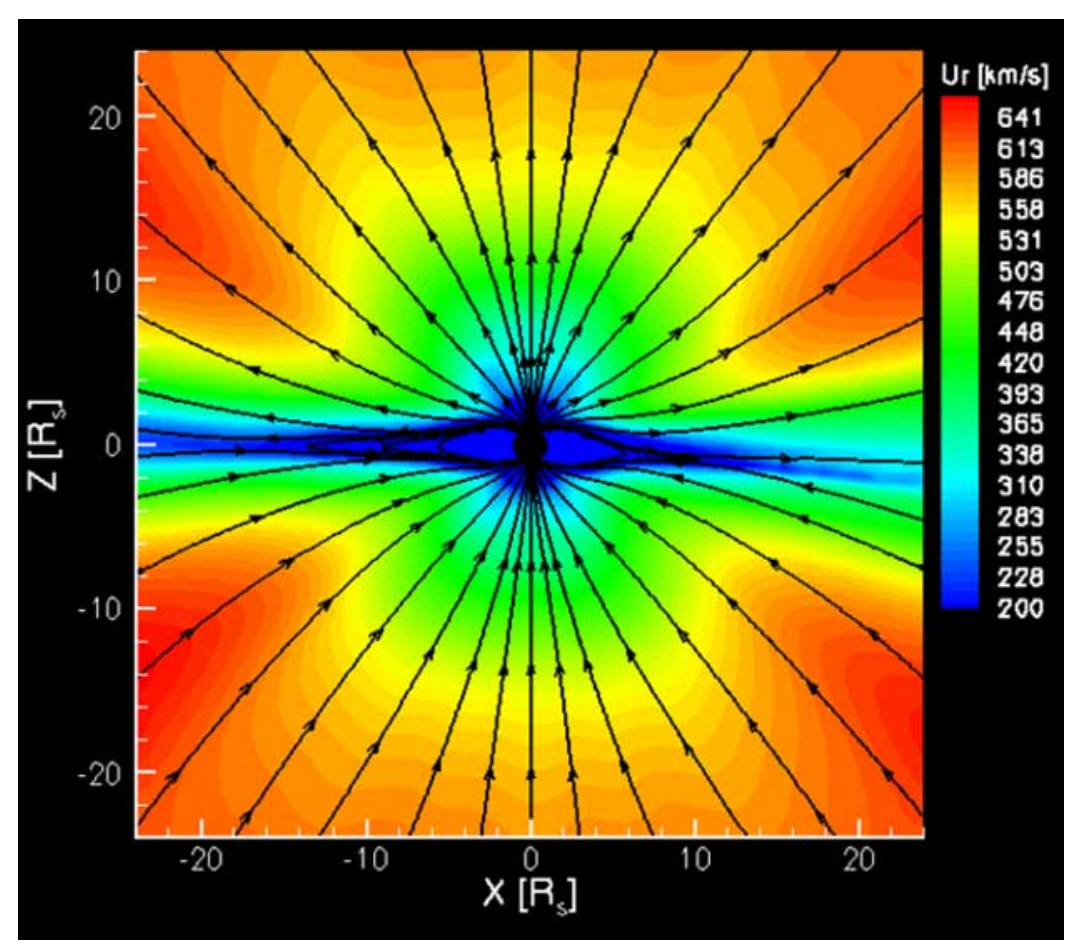

Figure 2. A cut in the steady state solution for the SC during CR 1922. Color contours represent the radial solar wind speed, and streamlines represent the magnetic field lines.

[14] Ideally, since the solar wind has to be super-Alfvénic at the SC-IH boundary, all the information should propagate outward. Therefore, it is sufficient to couple the modules only for a single iteration if the SC module is already in a steady state. In our model, the spherical SC-IH boundary is set at $r=17 R_{\odot}$, while the Cartesian outer boundary for SC is set at $x, y, z=24 R_{\odot}$. When the coupled mode is turned on, the inner boundary in $\mathrm{IH}$ is driven by the $\mathrm{SC}$ values at $r=17 R_{\odot}$, while the outer boundary conditions for $\mathrm{SC}$ are constrained by the IH values at the Cartesian distance of 24 $R_{\odot}$ from the center of the Sun. Therefore, the SC steady state solutions for the SC stand-alone run and coupled SC$\mathrm{IH}$ run are not identical. After obtaining a steady state in SC, we turn on the IH module for a coupled run, which is sufficient according to the steady state convergence parameter (see equation (A4) in Appendix A). We then turn off the SC module until a full steady state in $\mathrm{IH}$ is achieved. We refine the grid in the IH module to obtain a grid cell size of $r=0.2 R_{\odot}$ near the current sheet.

[15] In order to obtain a more physical solution for the heliospheric current sheet, we implement the Roe solver (RS) in our model [Sokolov et al., 2008]. The RS is the most exact and least diffusive Gudanov-type numerical solver. The use of the RS is practically equivalent to the use of another level of grid refinement. Therefore, it should better resolve the heliospheric current sheet, where magnetic reconnection occurs and the ideal MHD approximation suffers from numerical diffusion and magnetic reconnection.
[16] We run our model on the NASA ALTIX supercomputer with the use of 64 processors for each CR. With this amount of processors, it takes about 1 week of real time to obtain a steady state solution for 1 year.

\section{Results}

\subsection{Solar Minimum}

[17] We run the model for CRs 1916-1929 (November 1996 to November 1997). During this period the Sun was under solar minimum conditions, and the solar magnetic field configuration was dipolar.

[18] The relationship between the total magnetic flux predicted by the magnetogram-driven potential field model on one hand and the total magnetic flux observed at $1 \mathrm{AU}$ on the other hand is still under debate (e.g., Solar Heliospheric and Interplanetary Environment (SHINE) 20062007 workshops). Currently, the MDI data provider recommends multiplying the magnetogram by a factor of 1.8 (Y. Liu, private communication, 2007). However, for solar minimum conditions, we obtain a better result for the magnetic field with the use of a scaling factor of 2.5. For solar minimum, we use the base density value of $n_{0}=4.3 \times$ $10^{8} \mathrm{~cm}^{-3}$.

[19] Figure 2 shows a cut along the $y=0$ plane in the frame of reference rotating with the Sun, which represents the central meridian of CR 1922. Color contours represent the radial solar wind speed, while streamlines represent the magnetic field lines. We obtain a thin current sheet sur-

Figure 3. A comparison of the simulation results with ACE and Wind data at $1 \mathrm{AU}$. The comparisons are for (a) solar wind radial speed, (b) magnetic field strength, (c) number density, and (d) plasma temperature. 

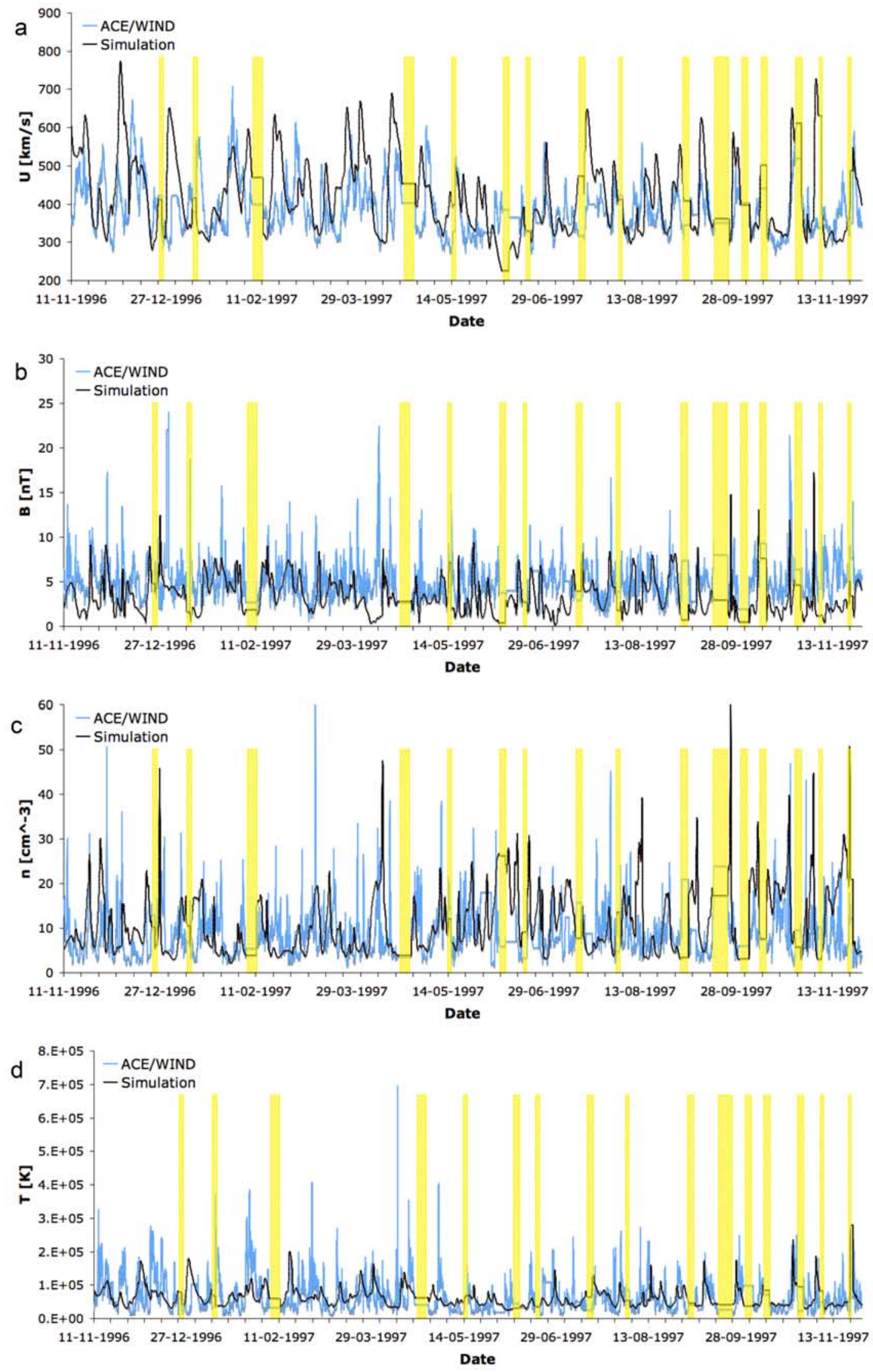

Figure 3 
Table 1. Statistical Analysis of Results for Solar Minimum Conditions

\begin{tabular}{ccc}
\hline Cross & Correlation & Normalized RMS \\
\hline$U_{r}$ & 0.82 & 27 \\
$N$ & 0.35 & 87 \\
$B$ & 0.4 & 59 \\
$T$ & 0.4 & 65 \\
\hline
\end{tabular}

rounded by slow solar wind speed of $300-400 \mathrm{~km} \mathrm{~s}^{-1}$, which extends up to $20^{\circ}$ above and below the current sheet.

[20] Figure 3 shows a comparison of the simulation results with $\mathrm{ACE}$ and Wind data at $1 \mathrm{AU}$. The comparisons are for solar wind radial speed (Figure 3a), magnetic field magnitude (Figure 3b), plasma number density (Figure 3c), and plasma temperature (Figure 3d). The blue line represents hourly averaged satellite data obtained from http:// cdaweb.gsfc.nasa.gov. The black line represents an extraction of the MHD variables along the satellite trajectory during the particular $\mathrm{CR}$ in the steady state simulation domain using the same time interval as the satellite data. The yellow bars mark the periods of time when CMEs were observed [ Cane and Richardson, 2003; Jian et al., 2006]. Since these are dynamical transients, the steady state model should not be expected to match the observations at these particular periods. Nevertheless, the model predicts rather well the periodic corotating interaction regions (CIRs). In order to avoid the effect of the CME transients on the statistics, we set both data and model values to zero during these periods.

[21] Table 1 shows statistical analysis of the results for solar minimum conditions. It shows the cross correlation (CC) between the model and the data and normalized root- mean-square (RMS) error. The statistical analysis has been done for all the points but the points defined as CME transients. The values are for the solar wind radial speed, $U_{r}$, plasma number density, $N$, magnetic field, $B$, and plasma temperature, $T$. The $\mathrm{CC}$ between the data and the model is very good $(0.8)$ for $U_{r}$ and reasonable (0.4) for the other variables. In particular, the model provides the correct magnitude of all the magnetohydrodynamic variables simultaneously, and the normalized RMS error is less than 1 (it is only $27 \%$ for $U_{r}$ ).

[22] Overall, the model predicts rather well interplanetary features (which can be obtained by a steady state model) such as CIRs and transients from fast to slow solar wind. However, in some cases, the inaccuracy in the solar wind speed shifts the features onset time earlier or later compared with the observations. The shifts are of the order of 1 day for a speed difference of $50 \mathrm{~km} \mathrm{~s}^{-1}$.

\subsection{Solar Maximum}

[23] We run the model for CRs 1972-1984 (January 2001 to January 2002). During this period the Sun was under solar maximum conditions, and the solar magnetic field was highly structured and was not dipolar any more.

[24] For solar maximum conditions, the magnetic flux obtained in the simulation is smaller than the observed flux at $1 \mathrm{AU}$ by a factor of at least 1.5 , even when the magnetogram scaling factor is 4 . For the solar maximum case, we multiply the model result for the magnetic field at 1 AU by a factor of 2, after using a scaling factor of 4 for the input MDI magnetogram. We discuss this magnetic flux issue in section 4. For solar maximum, we use base density value of $n_{0}=3.5 \times 10^{8} \mathrm{~cm}^{-3}$, which is slightly smaller than the base density used for solar minimum.

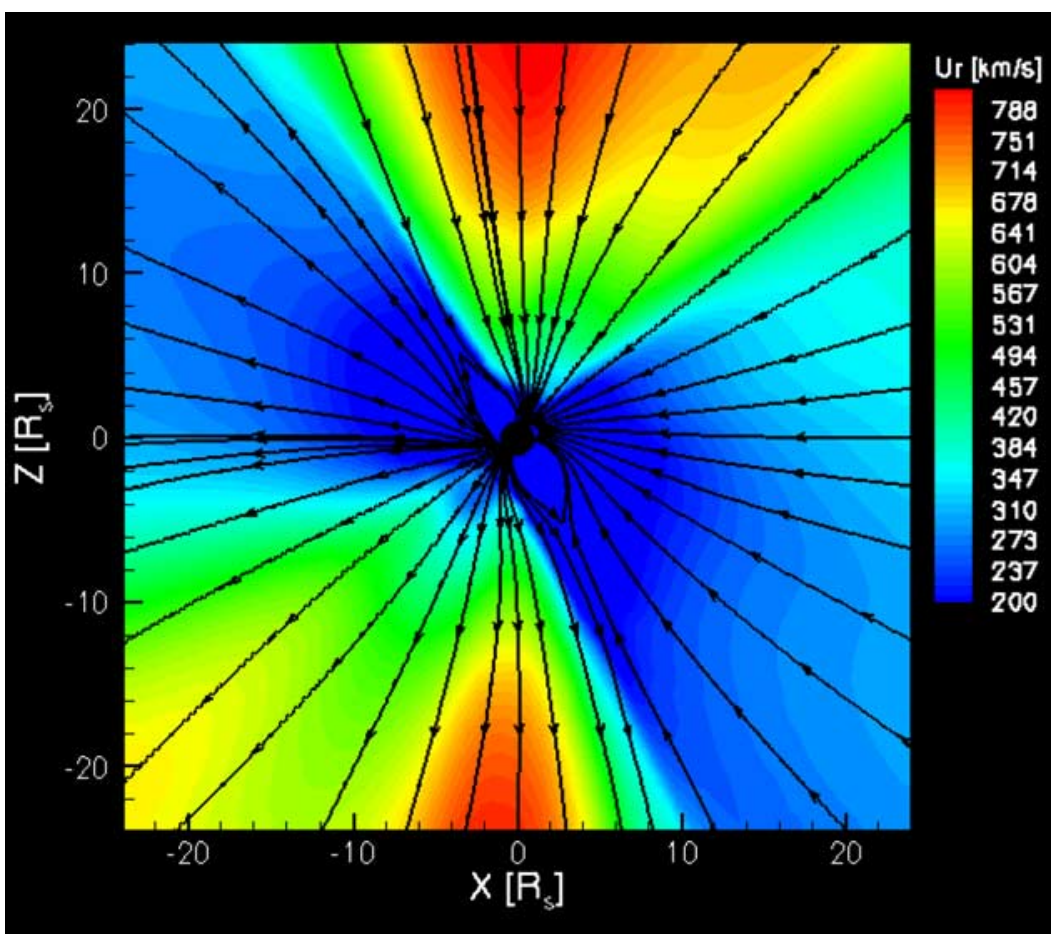

Figure 4. A cut in the steady state solution for the SC during CR 1973. Color contours represent the radial solar wind speed, and streamlines represent the magnetic field lines. 

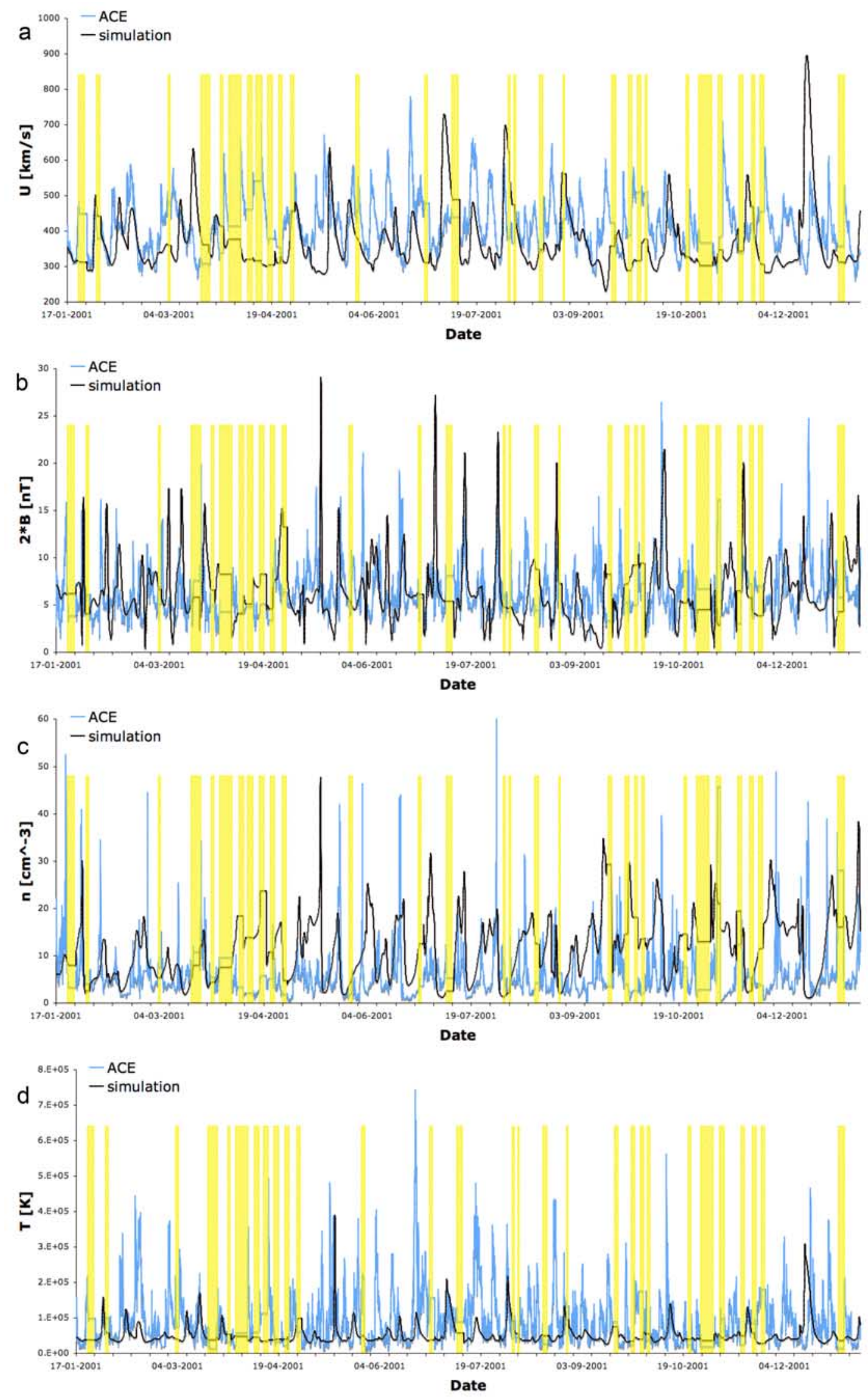

Figure 5. A comparison of the simulation results with ACE data at $1 \mathrm{AU}$. The comparisons are for (a) solar wind radial speed, (b) magnetic field strength, (c) number density, and (d) plasma temperature. 
Table 2. Statistical Analysis of Results for Solar Maximum Conditions

\begin{tabular}{lcc}
\hline Cross & Correlation & Normalized RMS \\
\hline$U_{r}$ & 0.79 & 29 \\
$N$ & 0.32 & 97 \\
$B$ & 0.5 & 62 \\
$T$ & 0.32 & 75 \\
\hline
\end{tabular}

[25] Figure 4 is similar to Figure 2 but for solar maximum conditions during CR 1973. One can see that the heliospheric current sheet is highly tilted and the slow solar wind extends to higher magnetic latitudes. Figure 5 shows a comparison of the model results with ACE data. Display settings are the same as Figure 3, and one can see that the amount of CME transients (yellow bars) is larger than in the case of solar minimum. Table 2 shows a statistical analysis of the solar maximum results. Overall, the statistics are similar to the solar minimum case, except for the fact that it has been done with the model results for the magnetic field multiplied by another factor of 2 .

\section{Discussion}

[26] In this paper, we attempt to validate our model by comparing its output with satellite data at 1 AU. Success was not guaranteed because the satellite measures the solar wind parameters along a particular trajectory, and the local values could be poorly predicted even by a global model, which is correct overall. The model predictions for the vicinity of the current sheet are especially subject to errors. With the model prediction for the sheet location offset by only a few grid points, the predicted solar wind speed would be much higher than observed. Nevertheless, the model predictions of the measurements are rather good, even for solar minimum when the satellite is located close to the current sheet. It is also important that we benefit from the reliability and simplicity of the WSA model without loss of accuracy.

[27] For solar maximum, the most meaningful result is the fact that the magnetic field magnitude at $1 \mathrm{AU}$ cannot be reproduced by the MHD model without using a very large scaling factor for the magnetogram data. This result is consistent with the idea that during solar maximum, about $30-50 \%$ of the observed heliospheric open flux originates from active regions and CMEs interchange reconnection [Fisk et al., 1999; Neugebauer et al., 2002; Wang and Sheeley, 2002; Schrijver and DeRosa, 2003; Owens and Crooker, 2006; Riley, 2007]. Although the prediction of the heliospheric open flux by the MHD model is not perfect, it is still useful in predicting a space weather event. This is due to the fact that the most crucial parameter for space weather event forecasting is the arrival time of the interplanetary shock and the orientation of $B_{z}$ (which we do not attempt to predict in this work). If the model can provide a reasonable prediction for the speed, density, and $B_{z}$, we should expect the magnitude of the background magnetic field to be less important.

[28] In this work, we adjust the model parameters for each type of solar condition in order to investigate whether this model can be operational. It appears that the results could be slightly improved by parameterizing the model for each particular CR, in particular, the solar wind speed and the arrival time of the periodic features such as CIRs.

\section{Conclusions}

[29] We have developed a three-dimensional numerical MHD model for the solar corona and the inner heliosphere. We have performed a long-term validation of the model for 1 year of solar minimum and 1 year of solar maximum using a comparison of the model results with satellite observations at $1 \mathrm{AU}$. Overall, the model is found to predict rather well the magnitude of the magnetohydrodynamical variables, as well as the quasi-steady corotating or recurrent features, in particular, for solar minimum conditions. For solar maximum conditions, the model predicts the solar wind speed, density, and temperature reasonably well on a large scale since transients are ignored. However, the model's prediction for the magnetic field magnitude at $1 \mathrm{AU}$ is still not reliable enough.

[30] Our model, as well as the WSA model, has a dependendency on the potential field approximation and the location of the source surface, which introduces another degree of freedom. Even though the model seems to be useful, we assume it will provide better results with the application of a more self-consistent solar wind heating mechanism such as Alfvén wave heating.

[31] In addition to space weather forecasting, our model can provide information about the large-scale structure and long-term evolution of the heliospheric magnetic field. This information can be useful to investigate large numbers of physical phenomena such as particle transport, evolution of the Parker spiral, interaction of the solar wind with the outer planets and comets, and the solar dynamo.

[32] In the context of space weather, we plan to investigate the model's capabilities to predict $B_{z}$ at $1 \mathrm{AU}$, which is a parameter of great importance for predicting geomagnetic activity.

\section{Appendix A: Local Time Stepping Algorithm and Its Convergence Rate}

[33] The absolute number of iterations, $n$, is not very meaningful by itself. To derive a relevant convergence parameter, $R$, for a particular grid, consider first the time accurate explicit scheme. In this case, the rate of the numerical solution convergence to a steady state is inversely proportional to the characteristic time:

$$
t_{c}=\int_{R=1}^{R_{\mathrm{OB}}} \frac{\Delta r}{C(x)},
$$

during which the physical perturbations propagate from, say, the inner boundary to the outer boundary (OB), with $x$ being the spatial scale and $C$ being the characteristic speed. Therefore, the proper convergence parameter is the ratio of the simulation time to the characteristic one:

$$
R=\frac{n \Delta t}{t_{c}}=\frac{n}{\sum_{n} \frac{1}{\operatorname{CFL}(n)}}
$$


where we represent the integral along the straight line as a sum by the cells it intersects, with the local CourantFriedrichs-Levy number CFL $(n)=C \Delta t / \Delta x$.

[34] For stability of the explicit scheme, we use CFL $\leq$ 0.8 . The values of the inverse to the CFL number in equation (A2) are close to unity only in the smallest cells near the Sun, where the speed $C$ has its maximum value $\left(C_{\max } \Delta t /(\Delta x)_{\min } \approx 0.8\right)$. To the contrary, at larger heliocentric distances, the inverse of the CFL number may be $2-$ 3 orders of magnitude larger $\left(\Delta x / \Delta x_{\min }\right)\left(C_{\max } / C(x)\right) \gg 1$, drastically degrading the convergence.

[35] That is why we use the local time stepping algorithm in simulating a steady state solution, which automatically maintains the local CFL number to be equal to 0.8 everywhere, with the choice of a local time step,

$$
\Delta t(x)=0.8 \frac{\Delta x}{C(x)} .
$$

[36] In this case the convergence parameter becomes

$$
R=\frac{0.8 n}{N_{x}},
$$

where $N_{x}$ is the typical number of cells (of any grid) that intersect with the line as described above.

[37] Acknowledgments. We would like to thank Yang Liu for providing the MDI magnetogram data and the reviewers for their comments. The research for this manuscript is supported by NSF ATM grant 0642309 , NASA grant NNX07AC16G, and AFOSR grant FA9550-07-1-0434 at the University of Michigan. The model development for the MHD turbulence and its role in the plasma heating is supported by the SMPD grant DEFC02-04ER54788. I.R. has been partially supported by NSF's SHINE grant ATM-0631790 and would like to acknowledge the limited support received from grant VU-01/06 with the Bulgarian NSF. I.S. is supported by contract F014254 between the JPL and the University of Michigan.

[38] Zuyin Pu thanks Dusan Odstrcil and Arcadi Usmanov for their assistance in evaluating this paper.

\section{References}

Arge, C. N., and V. J. Pizzo (2000), Improvement in the prediction of solar wind conditions using near-real time solar magnetic field updates, J. Geophys. Res., 105, 10,465-10,479.

Arge, C. N., J. G. Luhmann, D. Odstrcil, C. J. Schrijver, and Y. Li (2004), Stream structure and coronal sources of the solar wind during the May 12th, 1997 CME, J. Atmos. Sol. Terr. Phys., 66, 1295-1309.

Cane, H. V., and I. G. Richardson (2003), Interplanetary coronal mass ejections in the near-Earth solar wind during 1996-2002, J. Geophys. Res., 108(A4), 1156, doi:10.1029/2002JA009817.

Cohen, O., et al. (2007), A semi-empirical magnetohydrodynamical model of the solar wind, Astrophys. J., 645, L163-L166.

Cohen, O., I. V. Sokolov, I. I. Roussev, N. Lugaz, W. B. Manchester, T. I. Gombosi, and C. N. Arge (2008), Validation of a global 3-D heliospheric model with observations for the May 12, 1997 CME event, J. Atmos. Sol. Terr. Phys., doi:10.1016/j.jastp.2007.08.065, in press.

Fisk, L. A. (2003), Acceleration of the solar wind as a result of the reconnection of open magnetic flux with coronal loops, J. Geophys. Res., 108(A4), 1157, doi:10.1029/2002JA009284.

Fisk, L. A., N. A. Schwadron, and T. H. Zurbuchen (1999), Acceleration of the fast solar wind by the emergence of new magnetic flux, J. Geophys. Res., 104, 19,765-19,772.
Gleisner, H., and J. Watermann (2006), Concepts of medium-range (1-3 days) geomagnetic forecasting, Adv. Space Res., 37, 1116-1123.

Groth, C. P. T., D. L. DeZeeuw, T. I. Gombosi, and K. G. Powell (2000), Global three-dimensional MHD simulation of a space weather event: CME formation, interplanetary propagation, and interaction with the magnetosphere, J. Geophys. Res., 105, 25,053-25,078.

Jian, L., C. T. Russell, J. G. Luhmann, and R. M. Skoug (2006), Properties of interplanetary coronal mass ejections at one AU during 1995-2004, Sol. Phys., 239, 393-436.

Mikic, Z., J. A. Linker, D. D. Schnack, R. Lionello, and A. Tarditi (1999), Magnetohydrodynamic modeling of the global solar corona, Phys. Plasmas, 6, 2217-2224.

Neugebauer, M., P. C. Liewer, E. J. Smith, R. M. Skoug, and T. H. Zurbuchen (2002), Sources of the solar wind at solar activity maximum, J. Geophys. Res., 107(A12), 1488, doi:10.1029/2001JA000306.

Owens, M. J., and N. U. Crooker (2006), Coronal mass ejections and magnetic flux buildup in the heliosphere, J. Geophys. Res., 111, A10104, doi:10.1029/2006JA011641.

Phillips, J. L., et al. (1995), Ulysses solar wind plasma observations from pole to pole, Geophys. Res. Lett., 22, 3301-3304.

Powell, K. G., P. L. Roe, T. J. Linde, T. I. Gombosi, and D. L. De Zeeuw (1999), A solution-adaptive upwind scheme for ideal magnetohydrodynamics, J. Comput. Phys., 154, 284-309.

Riley, P. (2007), An alternative interpretation on the relationship between the inferred open solar flux and the interplanetary magnetic field, Astrophys. J., 667, L97-L100.

Roussev, I. I., T. I. Gombosi, I. V. Sokolov, M. Velli, W. Manchester, D. L. DeZeeuw, P. Liewer, G. Tóth, and J. Luhmann (2003), A threedimensional model of the solar wind incorporating solar magnetogram observations, Astrophys. J., 595, L57-L61.

Schrijver, C. J., and M. L. DeRosa (2003), Photospheric and heliospheric magnetic fields, Sol. Phys., 212, 165-200.

Sokolov, K. G., I. I. Powell, O. Cohen, and T. I. Gombosi (2008), Computational Magnetohydrodynamics, Based on Non-linear Solution of the Well-Posed Riemann Problem, Astron. Soc. Pac. Conf. Ser., Astron. Soc. of the Pac., San Francisco, Calif., in press.

Suess, S. T., A.-H. Wang, S. T. Wu, G. Poletto, and D. J. McComas (1999), A two-fluid, MHD coronal model, J. Geophys. Res., 104, 4697-4708.

Tóth, G., et al. (2005), Space Weather Modeling Framework: A new tool for the space science community, J. Geophys. Res., 110, A12226, doi:10.1029/2005JA011126.

Totten, T. L., J. W. Freeman, and S. Arya (1996), Application of the empirically derived polytropic index for the solar wind to models of solar wind propagation, J. Geophys. Res., 101, 15,629-15,636.

Usmanov, A. V. (1993), A global numerical 3-D MHD model of the solar wind, Sol. Phys., 146, 377-396.

Usmanov, A. V., and M. L. Goldstein (2003), A tilted-dipole MHD model of the solar corona and solar wind, J. Geophys. Res., 108(A9), 1354, doi:10.1029/2002JA009777.

Usmanov, A. V., M. L. Goldstein, B. P. Besser, and J. M. Fritzer (2000), A global MHD solar wind model with WKB Alfvén waves: Comparison with Ulysses data, J. Geophys. Res., 105, 12,675-12,696.

Wang, Y.-M., and N. R. Sheeley (1990), Solar wind speed and coronal fluxtube expansion, Astrophys. J., 355, 726-732.

Wang, Y. M., and N. R. Sheeley (2002), Sunspot activity and the long-term variations of the Sun's open magnetic flux, J. Geophys. Res., 107(A10), 1302, doi:10.1029/2001JA000500.

Wu, S. T., W. P. Guo, D. J. Michels, and L. F. Burlaga (1999), MHD description of the dynamical relationships between a flux rope, streamer, coronal mass ejection, and magnetic cloud: An analysis of the January 1997 Sun-Earth connection event, J. Geophys. Res., 104, 14,78914,802 .

O. Cohen, T. I Gombosi, and I. V. Sokolov, Department of Atmospheric, Oceanic, and Space Sciences, University of Michigan, 2455 Hayward Street, Ann Arbor, MI 48109-2143, USA. (oferc@umich.edu)

I. I. Roussev, Institute for Astronomy, University of Hawai'i at Manoa, 2680 Woodlawn Drive, Honolulu, HI 96822, USA. 